\title{
Metrics Beyond Hemoglobin A1C in Diabetes Management: Time in Range, Hypoglycemia, and Other Parameters
}

\author{
Lorena Alarcon-Casas Wright, MD, FACE, and Irl B. Hirsch, MD
}

\begin{abstract}
We review clinical instances in which $\mathrm{A} 1 \mathrm{C}$ should not be used and reflect on the use of other glucose metrics that can be used, in substitution of or in combination with A1C and SMBG, to tailor an individualized approach that will result in better outcomes and patient empowerment.
\end{abstract}

Keywords: Glucose metrics, Glycemic biomarkers, Continuous glucose monitoring, Flash-continuous glucose monitoring.

\section{Introduction}

$\mathbf{F}$ REQuent EVAluation AND PRECISE measurement of glucose control are a crucial part of optimal diabetes mellitus (DM) care. Hemoglobin A1C (A1C), along with self-monitoring of blood glucose (SMBG), is considered the gold standard treatment target for DM, due to the intervention studies in both type $1 \mathrm{DM}$ and type $2 \mathrm{DM}$, associating improved glucose control with a decreased risk of complications. ${ }^{1-3}$ With the advent of new technologies to assess glycemia, recent evidence linking hypoglycemia with adverse outcomes, and the increased knowledge on the limitations of $\mathrm{A} 1 \mathrm{C}$ and $\mathrm{SMBG}$, new metrics need to be incorporated, to better understand the dynamic nature of glucose, how to help patients achieve optimal control, reduce complications, and also to improve patient satisfaction by decreasing the burden of the interventions recommended. Multiple parameters of glucose control other than A1C have been proposed, potentially creating a burden for DM providers.

\section{Serum Biomarkers}

Glycemic biomarkers are surrogates to estimate the risk of chronic diabetes mellitus (DM) complications. They are used to determine whether a patient's average glucose control has been maintained at target range for a determined period of time, depending on the biomarker used. There are currently four clinical biomarkers: A1C, glycated proteins: fructosamine (FA) and glycated albumin (GA), the latter not clinically available in the United States and 1, 5-anhydroglucitol (1, 5-AG).

\section{Hemoglobin A1C}

A1C, in the setting of a normal hematological profile and in the nonpregnant population, reflects mean glycemia over the previous $8-12$ weeks. Its periodic monitoring is widely used and considered, along with self-monitoring of blood glucose (SMBG), the primary technique to assess DM control. ${ }^{4}$

Conditions that affect red blood cell (RBC) life span will impact its value, independent of glycemia. ${ }^{5}$ The degree of such impact is currently immeasurable and frequently not fully appreciated. Table 1 summarizes common conditions that affect the accuracy of A1C and other serum glucose biomarkers.

Higher A1C values have been described in minorities, mainly African Americans (AA), across different degrees of glucose tolerance status and independent of glycemia. ${ }^{6-8}$ Differences in the permeability of RBC to glucose, the enzymatic activity of the rate of production of $\mathrm{A} 1 \mathrm{C}$ are some of the mechanisms proposed. 9,10 The advent of new biomarkers that do not rely on RBC survival or intracellular glucose permeability has put into question whether $\mathrm{A} 1 \mathrm{C}$ is higher in 


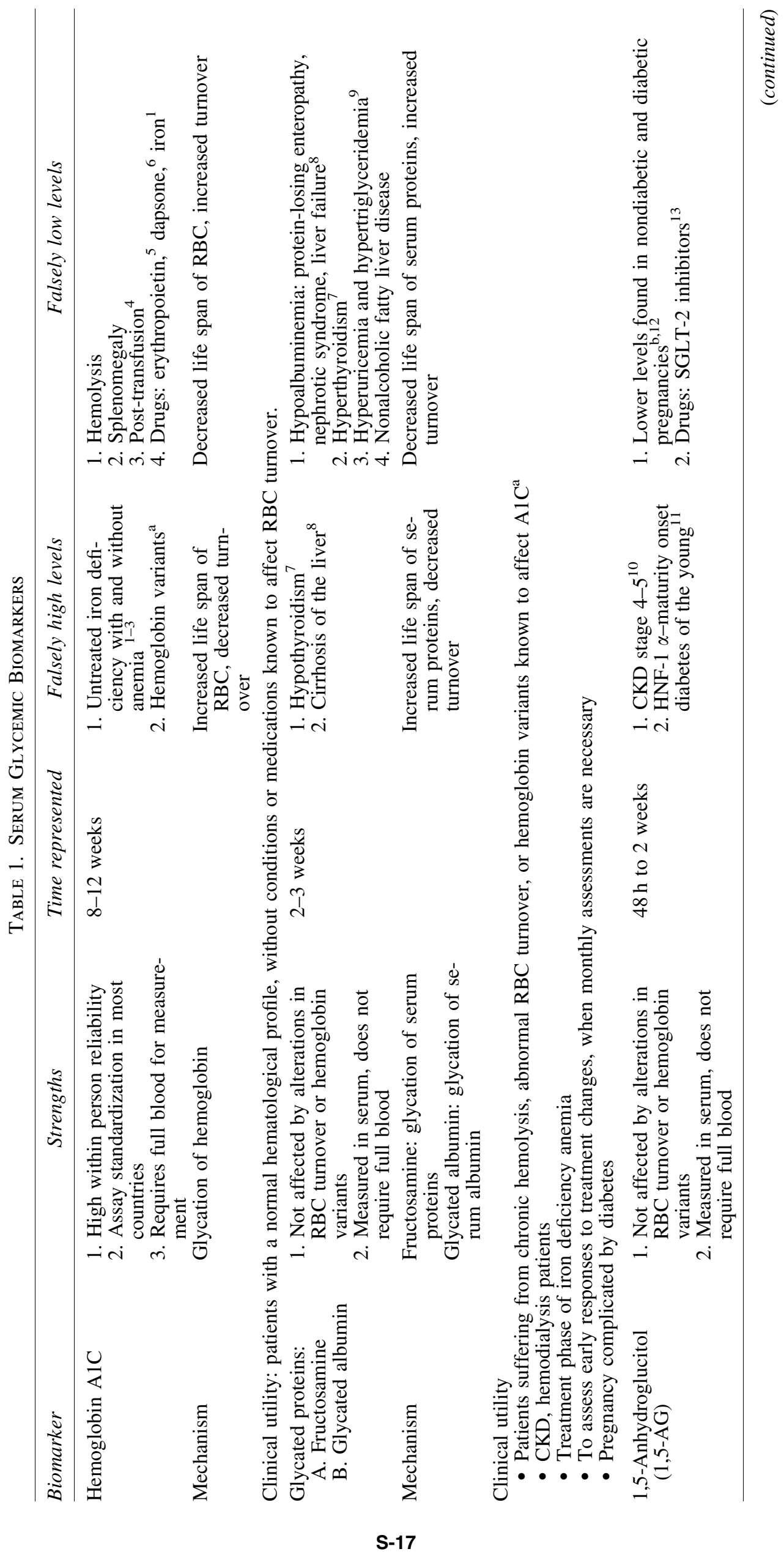




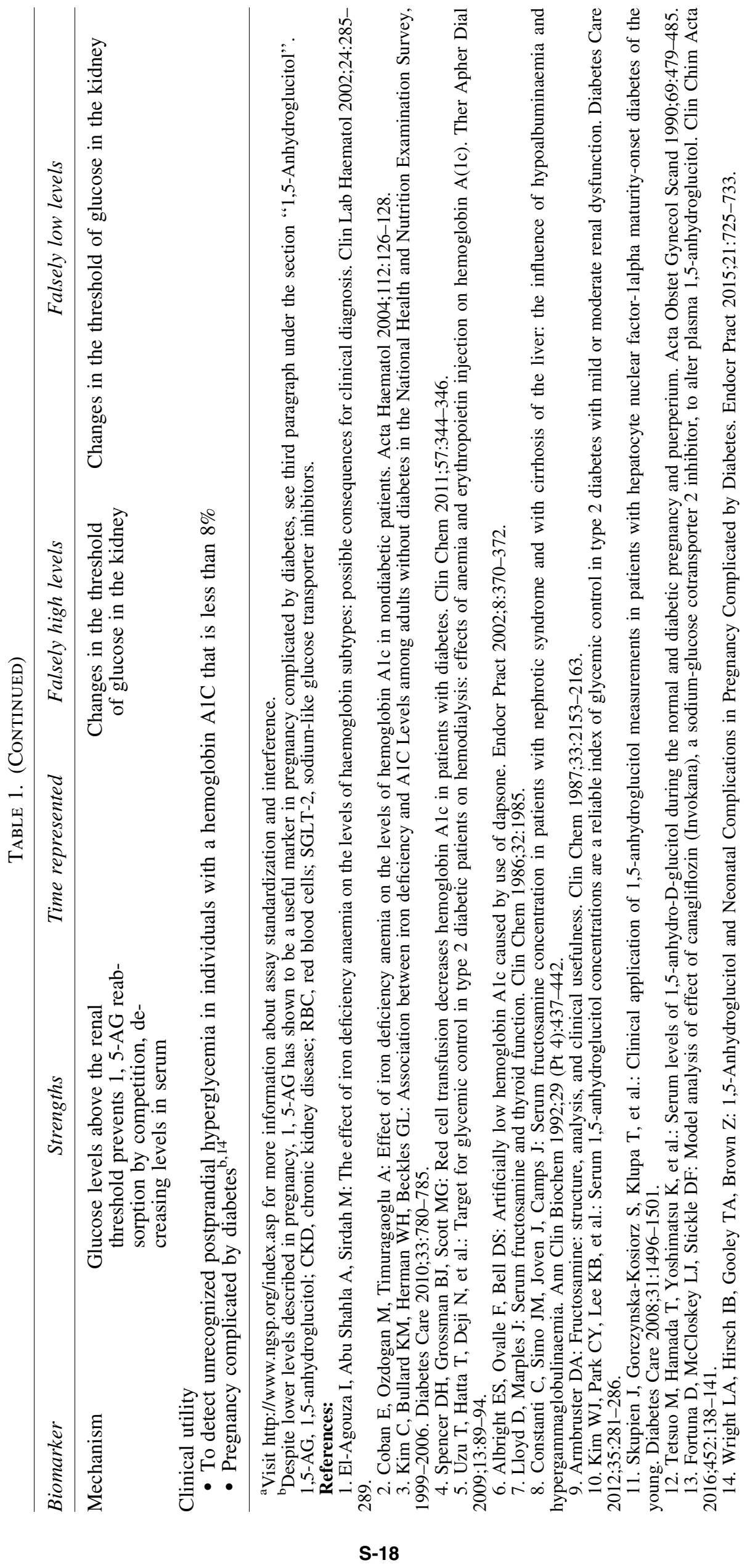


minorities independent of glycemia, suggesting such "discrepancies" in A1C may not always be independent of glucose. ${ }^{11}$ Nonetheless, in clinical practice, not frequently does the etiology of such differences often remain poorly understood (case 1).

\section{Glycated proteins}

In addition to hemoglobin, other proteins can become glycated. Glycated proteins are serum proteins that have permanently changed their composition when chronically exposed to glucose and turned into irreversible ketamines. FA refers to several proteins, with albumin accounting for $\sim 90 \%$; GA refers exclusively to albumin. ${ }^{12}$

Glycated proteins have been associated with micro- ${ }^{13-15}$ and macrovascular complications ${ }^{16-21}$ and mortality in people with DM. ${ }^{22}$ Several prospective studies have shown their utility for patients at risk of developing micro- and macrovascular complications. ${ }^{13,23,24}$

FA and GA are extracellular proteins that provide an index of average glucose over a period of 2-3 weeks, their average half-life, thus proposed as intermediate markers of glucose control. Their glycation rate is unaffected by RBC life span and therefore used in conditions in which A1C may not be a reliable marker, such as in hemodialysis patients, in whom several studies have shown superiority compared to $\mathrm{A} 1 \mathrm{C}^{25-27}$ and in whom both elevated FA and GA are risk factors for cardiovascular (CV) events, all-cause and CV mortality, independent of other confounding variables. ${ }^{28}$ Other scenarios of their utility include the evaluation of earlier response to treatment changes or in pregnancy complicated by diabetes. ${ }^{29}$

As the glycation rate depends on turnover of the protein of interest, any condition affecting its half-life will in turn influence the value independent of glucose control (Table 1).

Glycated proteins present with higher values relative to plasma glucose levels in patients with liver cirrhosis, and attributed to a couple of mechanisms, (1) prolonged half-life of serum albumin originating from reduced capacities of albumin synthesis in vivo ${ }^{30}$ and (2) increased immunoglobulin production in patients with cirrhosis, ${ }^{31}$ (globulins tend to increase in patients with cirrhosis and hepatitis, thought to be secondary to shunting of bacterial antigens in portal venous blood away from the liver to lymphoid tissue, which induces immunoglobulin production). ${ }^{32}$ However, as the synthetic function of the liver declines with worsening cirrhosis, albumin levels fall, and therefore in liver failure as albumin goes lower, glycated proteins will also decrease. GA is set lower in relation to plasma glucose levels in smokers, ${ }^{33}$ hyperuricemia patients, ${ }^{34}$ hypertriglyceridemia, ${ }^{35}$ and men with nonalcoholic fatty liver disease (NAFLD), ${ }^{36}$ the latter perhaps secondary to chronic microinflammation, where increase in albumin catabolism, particularly in obese subjects, shortens the half-life of albumin, decreasing glycated proteins relative to plasma glucose levels. ${ }^{37}$

FA concentrations are more likely to be influenced by the concentrations of protein and low-molecular-weight substances coexisting in the blood (e.g., bilirubin, uric acid), ${ }^{31}$ while GA reflects the proportion of GA to total serum albumin, expressed by a ratio (\%), as a result, the changes in serum protein concentration have less of an impact on GA than in FA. ${ }^{38,39}$

An important limitation to the use of glycated proteins is the lack of established clinical cut points, standardization, and data on frequency of measurements in clinical practice. Efforts have been made to estimate values similar to A1C levels, ${ }^{40-45}$ however, clinical targets continue to be unclear.

Glucose being the main energy source has many redundant regulation mechanisms, and its clinical interpretation requires the understanding that glycemia is a dynamic process, which is a limitation true to all glycated proteins, including A1C. Glycated protein values are "adynamic," making them crude measures of glycemia. Patients with similar A1C can have very different glucose patterns, rates of hypo- and hyperglycemia, and such fluctuations have an impact on A1C, depending on their severity, and unfortunately clinically unrecognizable by the value of the $\mathrm{A} 1 \mathrm{C}$ or marker alone, ${ }^{46}$ as such SMBG continues to be a tool to complement any of the available serum biomarkers.

The glycation gap hypothesis. The glycation of proteins is a continuous dynamic process that depends on many factors, many of them recognized (Table 1), however, others poorly understood, and perhaps not exclusively linked to average glycemia. The glycation gap (GG) refers to the difference between $\mathrm{A} 1 \mathrm{C}$ and the A1C predicted by the serum FA. The GG is negative if measured A1C is less than A1C predicted from FA and positive if measured $\mathrm{A} 1 \mathrm{C}$ is greater than predicted A1C. GG is zero when A1C and FA are concordant. A positive GG has been associated with the risk of microvascular complications in both type 1 and type $2 \mathrm{DM}$ (T2DM). ${ }^{24,47}$ Conversely, a negative GG has been described in patients with a lower risk of complications. ${ }^{48}$

\section{1,5-Anhydroglucitol}

1,5-AG is a monosaccharide, the 1-deoxy form of glucose. When glucose levels rise above the renal threshold for glucose, it will prevent $1,5-\mathrm{AG}$ reabsorption, leading to its excretion and thus decreasing serum levels. 1,5-AG reflects glucose control over the previous $48 \mathrm{~h}$ to 2 weeks, its concentration is mainly useful in detecting postprandial glucose (PPG), where lower levels result from glucose peaks above the renal threshold and particularly useful in patients with $\mathrm{A} 1 \mathrm{C}<8 \%$. ${ }^{49}$

$1,5-\mathrm{AG}$ was found to be negatively associated with longterm risk of microvascular outcomes, ${ }^{14,50,51}$ and an increased risk of CV disease ${ }^{52-54}$ and mortality in DM. ${ }^{53}$

The concentrations of 1,5-AG decrease as pregnancy progresses in both nondiabetic and diabetic subjects ${ }^{55}$; nonetheless, 1,5-AG has been found to be a useful marker in pregnancy complicated by DM, associated with mean glycemia, glycemic variability (GV), and glycemic exposure in females with T1D1 in whom glycemic control was assessed by a continuous glucose monitoring (CGM) system. ${ }^{56}$ Low levels are negatively associated with neonatal birth weight. ${ }^{56-58}$

The limitation of this biomarker derives mainly from renal function and factors that influence the renal threshold to glucose. ${ }^{59} 1,5-\mathrm{AG}$ has not been adequately studied in severe hyperglycemia and marked glycosuria $(\mathrm{A} 1 \mathrm{C}>10 \%) .{ }^{60}$ There are currently no guidelines of how often this biomarker should be obtained in clinical practice (Table 1).

\section{Self-Monitoring of Blood Glucose}

SMBG is a powerful tool available to patients to assess the effectiveness and safety of the regimen prescribed. For most $\mathrm{DM}$ patients on intensive insulin regimens, SMBG is 
recommended premeals, snacks, bedtime, occasionally after eating, pre-exercise, when suspicion of and postcorrection of hypoglycemia, and pretasks such as driving. ${ }^{4}$ Not surprisingly, there is a positive association of frequency of SMBG and improvements in glycemia. ${ }^{61}$

\section{Metrics derived from SMBG}

Fasting plasma glucose, PPG. The serum fasting plasma glucose (FPG) and 2-h PPG are recommended to diagnose DM. ${ }^{4}$ The 2-h PPG seems to diagnose more people with DM as early deterioration of glucose control is characterized by loss of PPG control. ${ }^{62}$ Both FPG and PPG provide a "snapshot" of glucose values, with relative contributions of these measures to A1C as A1C increases. ${ }^{63,64}$ Numerous studies link PPG with CV disease and CV events with a plausible pathophysiology ${ }^{65-68}$ and report that targeting PPG rather than FPG lowers CV risk, ${ }^{69}$ although others have reported no difference in $\mathrm{CV}$ event rates when targeting PPG. ${ }^{70}$ PPG is helpful to assess meal-induced glucose excursions and efficacy of DM treatment.

The glycemic risk assessment diabetes equation, average daily risk range. The glycemic risk assessment diabetes equation (GRADE) score refers to the degree of risk associated with a glucose profile. It is obtained from SMBG to quantify both hyper- and hypoglycemia by obtaining the percentage of time spent in specified given ranges (\% GRADE hypoglycemia, \% GRADE euglycemia, and $\%$ GRADE hyperglycemia). Values $<5$ correspond to euglycemia. $^{71}$

Average daily risk range (ADRR) is computed from 1 month of SMBG data, ideally three to five readings a day. The blood glucose data need to be mathematically transformed to give appropriate weight to hyper- and hypoglycemia and converted into their corresponding risk values. They are then implemented in a spreadsheet or software and based on the distribution of the ADRR, the values are stratified into risk categories: low risk $<20$; moderate risk, 20-40; and high risk, $>40 .^{72}$

In patients with type $1 \mathrm{DM}$ (T1DM), ADRR has shown to correlate positively with insulin sensitivity and negatively with the release of epinephrine, postulating that higher insulin sensitivity and lower epinephrine response during hypoglycemia are associated with higher $\mathrm{GV} .^{73}$ It has been associated with A1C and negatively associated with Cpeptide levels, suggesting that decreased $\beta$ cell function is associated with higher GV. ${ }^{74}$ In adults with T2DM, ADRR scores from CGM correlated with time spent below the target glucose range. ${ }^{75}$ Several studies have found ADRR to be relatively insensitive to treatment change when using realtime CGM. ADRR appears to be a good marker/predictor of extreme glucose values but a conservative measure of $\mathrm{GV}$, as summarized by Patton et al. ${ }^{76}$

\section{Continuous Glucose Monitoring}

Dramatic changes driven by technical advances in testing continue to take place; in turn, the results of more advanced monitoring options are increasing the evidence that the chronic complications of DM are not only the result of chronic hyperglycemia but also GV and hypoglycemia. CGM has made it apparent that periodic previous met- rics are insufficient to optimally manage glycemia in DM. CGM overcomes the limitations many of the traditional metrics pose.

Several CGM systems are currently available and can be divided into retrospective, real-time, or flash-monitoring systems.

\section{Professional retrospective CGM}

Professional retrospective CGM (PCGM) refers to the use of a subcutaneous CGM that the patient wears but it is blind to the results until the provider downloads and reviews the data, with the goal of adjusting insulin doses or assessing patterns and providing education to modify patient's behaviors. In the outpatient setting, it is a tool to identify patterns and/or otherwise unrecognized reasons of poor glucose control; and in patients in whom personal CGM is not an option.

Studies have looked at PCGM utility on improving A1C, and results have been conflicting. There was no reported improvement in A1C, 7 months after the intervention in a retrospective study that included 102 patients with $\mathrm{T} 1$ and T2DM. ${ }^{77}$ Others have reported an improvement on A1C in hyperglycemic patients with $\mathrm{T}_{1 \mathrm{DM}}{ }^{78}$ and both $\mathrm{T} 1$ and T2DM, ${ }^{78,79}$ and in patients with T2DM, having mainly hypoglycemia ${ }^{78}$; in this same study, there was no change in A1C in patients classified as having fluctuating glucose levels with either form of DM.$^{79}$ Durability of glucose control after PCGM revealed that the improvement at 3-5 months after PCGM was lost after 1 year. ${ }^{78}$ An improvement in selfreported hypoglycemia has been observed. ${ }^{78,79}$

There are no randomized control trials or clinical guidelines on the indications for this technology. Previous retrospective studies have included hypo- and hyperglycemia, ${ }^{79}$ in addition to $\mathrm{GV}^{78}$ as reasons for providers to prescribe this technology to patients. Our group identified GV and hyperglycemia as the most common indications for ordering a PCGM in an academic setting, and described improvements in A1C without a significant change in frequency of SMBG or mean glucose and no difference in self-reported hypoglycemia after PCGM. Not surprisingly, in patients who performed more frequent SMBG, change in A1C was more significant. ${ }^{80}$

\section{Personal CGM}

In contrast with a static picture (six to eight blood glucose measurements a day), the real-time nature of this monitoring tool allows patients to intervene when glucose values change rapidly and prevent glucose excursions and exposure to hyper- and/or hypoglycemia. Personal CGM allows retrospective analysis of complete profiles, by patients at home and/or by providers in clinic or remotely, facilitating an individualized approach to DM.

Improved glycemic control, hypoglycemia rates, and patient satisfaction have been demonstrated with CGM use; despite these advantages, CGM continues to be underutilized. The cost of the device and supplies, lack of or limited insurance, and patient and provider perceptions play a role.

Clinician's lack of familiarity pose a barrier at several stages of the process: limited knowledge on candidacy for CGM, technology, software needed in clinic and time to download, interpret, and provide education and feedback to patients. 
In 2013, Recommendations for Standardizing Glucose Reporting and Analysis to Optimize Clinical Decision Making in Diabetes: The Ambulatory Glucose Profile (AGP) were published; in recognizing that the widespread application of CGM was hampered by the lack of accepted measures for assessment and reporting of glucose profiles/data, likely contributing to the clinicians' reluctance to incorporate this tool in clinical practice. ${ }^{81}$

Metrics derived from CGM. The key metrics identified as part of the AGP were target range, glucose exposure, GV, hypoglycemia, and hyperglycemia.

Target range and time in range. Target range and time in range can be expressed either as "\% of glucose readings" or "hours per day." The proposed target range of 70-180 mg/ $\mathrm{dL}$ was considered acceptable for clinical practice, as it has been observed that if $50 \%$ of the SMBG readings are in such range, $\mathrm{A} 1 \mathrm{C}$ would be around $7 \% .^{82}$

Glucose exposure. Glucose exposure refers to the mean or average glucose, and a metric clinicians and patients are familiar with. Mean glucose exposure for specific time periods during CGM (e.g., overnight, fasting, 2-4h postprandial) is helpful in evaluating the effects of food, exercise, or insulin and easy to implement in clinical practice.

Indices of $G V . \mathrm{GV}$ has been of interest to DM clinicians and researchers for decades. Many studies have strongly suggested that GV (the acute excursions of glucose around a mean value, that is, hyperglycemic glucose fluctuations but also hypoglycemic exposure around mean glucose) may be a significant risk factor for microvascular complications, and that it may help to explain why some patients develop microvascular complications and others having the same A1C do not.

The finding by the Diabetes Complications and Control Trial (DCCT) that patients with same A1C levels in the intensive and conventional arms of therapy had differing rates of microvascular complications ${ }^{83}$ was a strong stimulus in the diabetes scientific community to search deeper into glycemic risk factors other than A1C as contributors of DM complications, namely GV. The subject continues to be controversial, however, it is accepted that $\mathrm{GV}$ is a strong predictor of hypoglycemia, ${ }^{84-86}$ leading to poor glucose control, which in turn results in poor patient satisfaction and may increase the risk of DM burden and poor compliance. Minimizing GV is necessary to achieve glucose stability and decrease the risk of hypoglycemia. ${ }^{87,88}$

To standardize measures of glycemia and for the ease of use, familiarity, and correlation with other factors of glycemic control, three measures of GV were proposed: standard deviation around the mean glucose (SD), coefficient of variation $(C V)$, and interquartile range (IQR). ${ }^{81}$

Standard deviation around the mean glucose. The metric most commonly used and understood for assessing and reporting GV is SD. SD of blood glucose was a predictor of the prevalence of peripheral neuropathy ${ }^{89}$ and is associated with microvascular complications ${ }^{90}$ and subclinical atherosclerosis in T1DM. ${ }^{91}$

Analysis of 30 measures of quality of glycemic control and variability from patients with $\mathrm{T} 1$ and $\mathrm{T} 2 \mathrm{DM}$ receiving insulin during a 1-week period of using CGM concluded that most of the GV measures were highly correlated with the overall $\mathrm{SD},{ }^{87}$ somewhat validating its clinical use. Criticisms to SD arise from the fact that often the glucose data are not normally distributed around the mean, and that the reliability of SD is influenced by the distribution of the data; as such, indexes with the use of formulas or equations have been developed, ${ }^{72,92}$ however, impractical to implement in busy clinical settings.

A ratio of the mean glucose to SD has been proposed, with values of 3 considered good and values of 2 considered poor. ${ }^{93}$ The results of Rodbard et al. were in agreement with this easy practical calculation. ${ }^{87}$ This easy formula has become the standard in our clinic, only when the mean is between 120 and $180 \mathrm{mg} / \mathrm{dL}$. With a ratio of 3 and the mean glucose $<120 \mathrm{mg} / \mathrm{dL}$, too much hypoglycemia is present, while with more severe mean hyperglycemia, overall control is obviously poor. ${ }^{87}$

Coefficient of variation. Derived from SD $(100 \times \mathrm{SD} / \mathrm{mean}$ of observations). The relative constancy of its percentiles irrespective of A1C or mean glucose levels, preventing a strong dependency of SD and other measures of mean glucose values, characterizes $C V$ as a good parameter of $\mathrm{GV}$. A good metric for research purposes, however, not easily displayed and therefore less helpful as part of the CGM clinical view.

If there is a low mean glucose and a large SD and hence a large $\% C V$, the risk of hypoglycemia will be high. In contrast, if both the mean and SD are high but with a low $\% C V$, the risk of hypoglycemia will be relatively low. Similarly for the risk of hyperglycemia, a high mean will generate a high frequency of hyperglycemia that is relatively insensitive to the magnitude of the SD. A lower mean could generate a high risk of hyperglycemia if the SD is large but not if the SD were small.

The correlation of $\% C V$ with risk of hypoglycemia has been observed, ${ }^{85}$ enhancing its utility as a $\mathrm{GV}$ parameter. ${ }^{94} \mathrm{It}$ is hopeful that $\% C V$, which has become the standard for measurement of GV in clinical research, will become available for clinical use in the future.

Interquartile range. IQR takes the difference between the 75 th and 25 th percentiles of glucose values, and that $50 \%$ of glucose values are the IQR. It has the advantage of being easily recognized and not dependent on the assumption of normal distribution. It allows easy visibility of the time of day or relationship to a meal or medication that there is high $\mathrm{GV}$, which may need clinical attention. ${ }^{95,96}$

Hypoglycemia. Hypoglycemia is the major barrier in patients with DM and the limiting factor to achieve euglycemia. ${ }^{97}$ Studies have linked hypoglycemia with excessive morbidity and mortality. ${ }^{98-100}$ In light of the importance of reduction of hypoglycemia, efforts have been made for a consensus on reporting its frequency and severity. ${ }^{101}$ Consensus from the AGP to report hypoglycemia in users of CGM categorized it corresponding to glucose levels: low if glucose is $<70 \mathrm{mg} / \mathrm{dL}$; very low if $<60 \mathrm{mg} / \mathrm{dL}$, and dangerously low if $<50 \mathrm{mg} / \mathrm{dL}$. The percentage of glucose values below these thresholds and time in each range, as well as number of episodes (defined as at least 10 consecutive min below the criteria) of each range, were also recommended to 


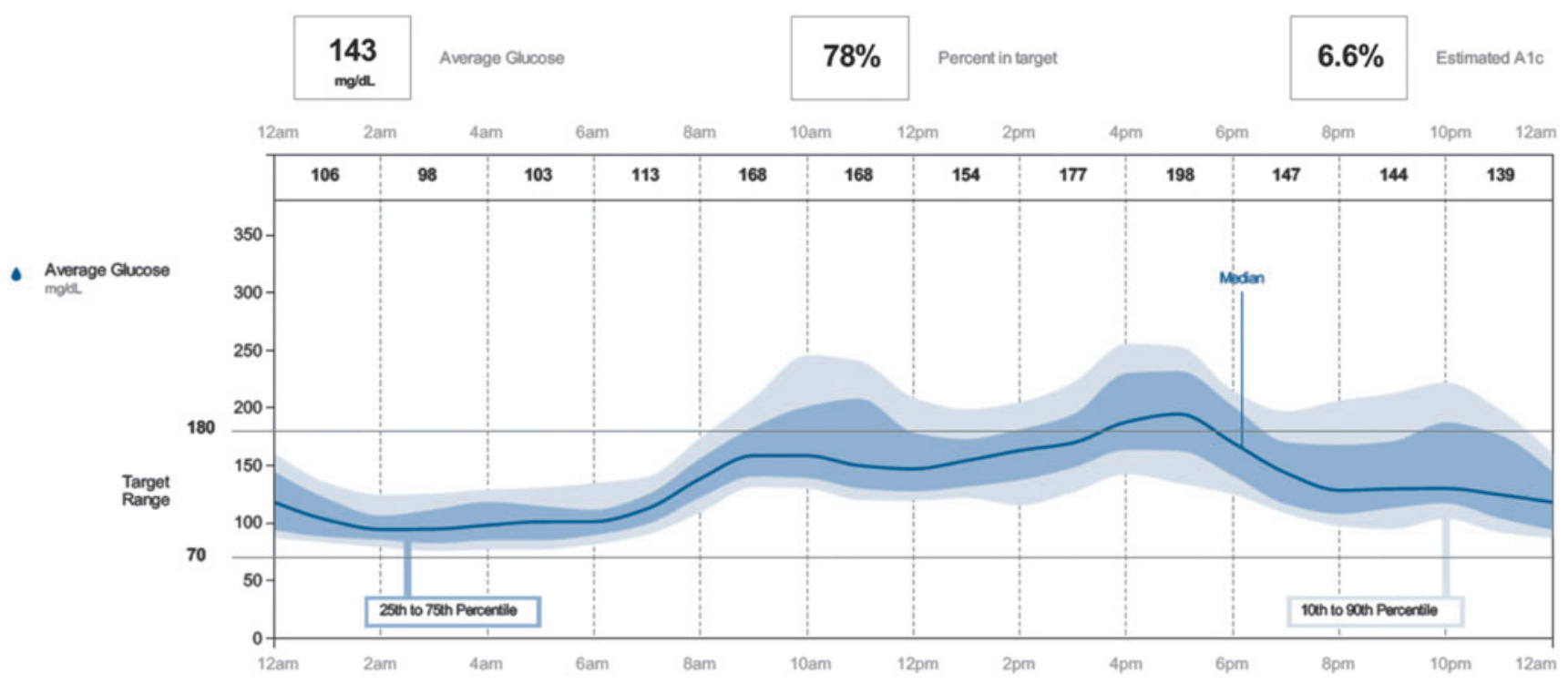

FIG. 1. FCGM in a 60-year-old African American woman with a falsely elevated A1C of $8.1 \%$.

be reported. A Joint Position Statement of the American Diabetes Association (ADA) and the European Association for the Study of Diabetes proposed the following glucose levels when reporting hypoglycemia in clinical trials: level 1: glucose alert value of $70 \mathrm{mg} / \mathrm{dL}$ or less; level 2: a glucose level $<54 \mathrm{mg} / \mathrm{dL}$, a sufficiently low value to indicate serious, clinically important hypoglycemia, and level 3: severe cognitive impairment requiring external assistance for recovery. 4,102

Hyperglycemia. Levels of severity above the upper range of the target of $180 \mathrm{mg} / \mathrm{dL}$ for glucose are recommended as follows: high $>180 \mathrm{mg} / \mathrm{dL}$; very high $>250 \mathrm{mg} / \mathrm{dL}$, and dangerously high $>400 \mathrm{mg} / \mathrm{dL}$. As with hypoglycemia, a clinically based category of severe hyperglycemia, that is, diabetic ketoacidosis, was recommended to advance surveillance from the patient's part and the clinical team (i.e., troubleshooting technical issues with pump therapy, hydration, and more frequent insulin correction) to prevent escalation of ketonemia and hospitalizations.

\section{Flash Glucose-Sensing Technology}

The flash continuous glucose monitoring (FCGM) system technology is a factory-calibrated interstitial glucose monitoring system, currently available as a professional, blind to the patient option (FreeStyle ${ }^{\circledR}$ Libre $^{\mathrm{TM}}$ Pro), and also as a personal monitoring system intended to substitute SMBG. FCGM uses a wired glucose oxidase enzyme coimmobilized on an electrochemical sensor, worn on the back of the arm for up to 14 days. The personal FCGM is currently not available in the United States. Patients obtain a real-time reading on demand as often as every minute by scanning the sensor with a reader. Data are transferred from the sensor to the reader memory and stored automatically every $15 \mathrm{~min}$ allowing to show trends for the previous $8 \mathrm{~h}$ on the screen, rate, and direction of glucose. The FCGM has no alarms. The data can be uploaded to obtain summary reports, for personal review or in clinic by DM providers.
FCGM is accurate, with reported overall mean absolute relative difference (MARD) of $11.4 \%$ for sensor results and stable over 14 days of use when compared with capillary BG reference values, and unaffected by body mass index, age, type of DM, clinical site, insulin administration, or A1C. ${ }^{103}$ The use of FCGM has been associated with improvement in glucose control in both uncontrolled T2 and T1DM, and maintained for up to 24 weeks of using the device. ${ }^{104}$ In wellcontrolled patients with T1DM, the use of FCGM reduced the time spent in hypoglycemia by $38 \%$ at 6 months (intervention group) versus controls (SMBG). ${ }^{105}$ The benefits in reduction of hypoglycemia by the use of FCGM have subsequently been reproduced in patients with both T1 and T2DM on treatment with insulin. In younger than 65-year-old T2DM patients, FCGM decreases A1C, while also reducing time in hypoglycemia by $43 \%, 53 \%$, and $64 \%$ in ranges of $<70 \mathrm{mg} / \mathrm{dL}$, $<55 \mathrm{mg} / \mathrm{dL}$, and $<45 \mathrm{mg} / \mathrm{dL}$, respectively, when compared to the SMBG group. Nocturnal and daytime hypoglycemia decreased by $54 \%$ and $31 \%$, respectively. Interestingly in this study, of the 224 randomized participants, in those aged $>65$ years, FCGM did not decrease A1C, compared to the control group (SMBG), nonetheless, time in hypoglycemia was reduced by $56 \%$ in FCGM users. ${ }^{106}$

A direct head-to-head comparison of CGM (Dexcom G4 Platinum) and FCGM showed that glucose profiles and MARD in outpatients with T1DM for up to 14 days were similar between the two sensors and no significant difference was detected in the estimation of clinical diagnostic parameters. ${ }^{107}$ FCGM has been positively associated with treatment satisfaction and measures of quality of life. ${ }^{105,106,108}$

Figure 1 gives a good example on how FCGM can impact DM therapy. In a 60-year-old AA woman with T2DM on metformin and sulfonylurea, A1C was $8.1 \%$ and testing $2 \times /$ day her mean glucose was $132 \mathrm{mg} / \mathrm{dL}$. This was suspicious for an inaccurate higher than predicted by SMBG A1C. FCGM showed a mean of $143 \mathrm{mg} / \mathrm{dL}$ and an estimated A1C of $6.6 \%$. This degree of difference is not uncommon in AA, the FCGM confirmed the discrepancy between her glucose and her A1C. 


\section{Conclusions}

A1C continues to be the gold standard for assessing glucose control in patients with DM, however, it has become evident that in clinical practice, many instances exist where A1C will not be a true reflection of average glucose, because its accuracy has been compromised by a variable affecting RBC survival and/or because of a GG. At the present time, all currently available glycemic biomarkers have advantages and limitations and it remains unclear which marker or combination of them may have the best relationship to complications for different populations of patients. For an individual patient, all of our biomarkers, including A1C, give a crude evaluation of glucose control.

Clinicians managing patients with DM should become familiar with a more expanded definition of optimal glucose control that includes not only A1C (when accurate) but also a combination of other metrics that reflect more realistically the dynamic nature of glucose control, by taking into consideration the period examined, limitations of each metric selected, comorbidities, medications (insulin, oral hypoglycemic), and also the feasibility and burden of the intervention recommended (SMBG, CGM, FCGM).

Recognizing the interplay between glucose control and behavior in DM, personal CGM and FCGM arise as an option to document and to intervene in the prevention of many components within the dysglycemia frame, not only reflected by average glucose or A1C but also by fluctuations and their potential short- and long-term risks. Personal CGM is now another option for many patients to better document if there indeed is a GG, and even if not, how more informative decisions can be made. CGM reported in a standardized way has the potential to help clinicians empower patients and decrease the burden of living with DM and its complications.

\section{Author Disclosure Statement}

Dr. Lorena Alarcon-Casas Wright has no conflicts of interest. Dr. Irl B. Hirsch is a consultant for Abbott Diabetes Care, Intarcia, Roche, and Valeritas.

\section{References}

1. The effect of intensive treatment of diabetes on the development and progression of long-term complications in insulin-dependent diabetes mellitus. The Diabetes Control and Complications Trial Research Group. N Engl J Med 1993;329:977-986.

2. Intensive blood-glucose control with sulphonylureas or insulin compared with conventional treatment and risk of complications in patients with type 2 diabetes (UKPDS 33). UK Prospective Diabetes Study (UKPDS) Group. Lancet 1998;352:837-853.

3. Effect of intensive blood-glucose control with metformin on complications in overweight patients with type 2 diabetes (UKPDS 34). UK Prospective Diabetes Study (UKPDS) Group. Lancet 1998;352:854-865.

4. Standards of Medical Care in Diabetes-2017: Summary of revisions. Diabetes Care 2017;40(Suppl 1):S4-S5.

5. Sacks DB: Hemoglobin A1c in diabetes: panacea or pointless? Diabetes 2013;62:41-43.

6. Herman WH, Ma Y, Uwaifo G, et al.: Differences in A1C by race and ethnicity among patients with impaired glu- cose tolerance in the Diabetes Prevention Program. Diabetes Care 2007;30:2453-2457.

7. Saydah S, Cowie C, Eberhardt MS, et al.: Race and ethnic differences in glycemic control among adults with diagnosed diabetes in the United States. Ethn Dis 2007;17: 529-535.

8. Herman WH, Dungan KM, Wolffenbuttel BH, et al.: Racial and ethnic differences in mean plasma glucose, hemoglobin A1c, and 1,5-anhydroglucitol in over 2000 patients with type 2 diabetes. J Clin Endocrinol Metab 2009;94:1689-1694.

9. Yudkin JS, Forrest RD, Jackson CA, et al.: Unexplained variability of glycated haemoglobin in non-diabetic subjects not related to glycaemia. Diabetologia 1990;33:208215.

10. Khera PK, Joiner $\mathrm{CH}$, Carruthers A, et al.: Evidence for interindividual heterogeneity in the glucose gradient across the human red blood cell membrane and its relationship to hemoglobin glycation. Diabetes 2008;57:2445-2452.

11. Selvin E, Francis LM, Ballantyne CM, et al.: Nontraditional markers of glycemia: associations with microvascular conditions. Diabetes Care 2011;34:960-967.

12. Armbruster DA: Fructosamine: structure, analysis, and clinical usefulness. Clin Chem 1987;33:2153-2163.

13. Selvin E, Rawlings AM, Grams M, et al.: Fructosamine and glycated albumin for risk stratification and prediction of incident diabetes and microvascular complications: a prospective cohort analysis of the Atherosclerosis Risk in Communities (ARIC) study. Lancet Diabetes Endocrinol 2014;2:279-288.

14. Mukai N, Yasuda M, Ninomiya T, et al.: Thresholds of various glycemic measures for diagnosing diabetes based on prevalence of retinopathy in community-dwelling Japanese subjects: the Hisayama Study. Cardiovasc Diabetol 2014;13:45.

15. Kondaveeti SB, Kumaraswamy D, Mishra S, Kumar A, Shaker IA: Evaluation of glycated albumin and microalbuminuria as early risk markers of nephropathy in type 2 diabetes mellitus. J Clin Diagn Res 2013;7:1280-1283.

16. Furusyo N, Koga T, Ai M, et al.: Plasma glycated albumin level and atherosclerosis: results from the Kyushu and Okinawa Population Study (KOPS). Int J Cardiol 2013; 167:2066-2072.

17. Lu L, Pu LJ, Xu XW, et al.: Association of serum levels of glycated albumin, C-reactive protein and tumor necrosis factor-alpha with the severity of coronary artery disease and renal impairment in patients with type 2 diabetes mellitus. Clin Biochem 2007;40:810-816.

18. Jin C, Lu L, Zhang RY, et al.: Association of serum glycated albumin, C-reactive protein and ICAM-1 levels with diffuse coronary artery disease in patients with type 2 diabetes mellitus. Clin Chim Acta 2009;408:45-49.

19. Lu L, Pu LJ, Zhang Q, et al.: Increased glycated albumin and decreased esRAGE levels are related to angiographic severity and extent of coronary artery disease in patients with type 2 diabetes. Atherosclerosis 2009;206: 540-545.

20. Song SO, Kim KJ, Lee BW, et al.: Serum glycated albumin predicts the progression of carotid arterial atherosclerosis. Atherosclerosis 2012;225:450-455.

21. Shen Y, Pu LJ, Lu L, et al.: Glycated albumin is superior to hemoglobin A1c for evaluating the presence and severity of coronary artery disease in type 2 diabetic patients. Cardiology 2012;123:84-90. 
22. Selvin E, Rawlings AM, Lutsey PL, et al.: Fructosamine and glycated albumin and the risk of cardiovascular outcomes and death. Circulation 2015;132:269-277.

23. Nathan DM, McGee P, Steffes MW, Lachin JM: Relationship of glycated albumin to blood glucose and HbA1c values and to retinopathy, nephropathy, and cardiovascular outcomes in the DCCT/EDIC study. Diabetes 2014;63:282-290.

24. Cohen RM, LeCaire TJ, Lindsell CJ, et al.: Relationship of prospective $\mathrm{GHb}$ to glycated serum proteins in incident diabetic retinopathy: implications of the glycation gap for mechanism of risk prediction. Diabetes Care 2008;31: 151-153.

25. Inaba M, Okuno S, Kumeda Y, et al.: Glycated albumin is a better glycemic indicator than glycated hemoglobin values in hemodialysis patients with diabetes: effect of anemia and erythropoietin injection. J Am Soc Nephrol 2007;18:896-903.

26. Vos FE, Schollum JB, Coulter CV, et al.: Assessment of markers of glycaemic control in diabetic patients with chronic kidney disease using continuous glucose monitoring. Nephrology (Carlton) 2012;17:182-188.

27. Freedman BI, Shihabi ZK, Andries L, et al.: Relationship between assays of glycemia in diabetic subjects with advanced chronic kidney disease. Am J Nephrol 2010;31: 375-379.

28. Shafi T, Sozio SM, Plantinga LC, et al.: Serum fructosamine and glycated albumin and risk of mortality and clinical outcomes in hemodialysis patients. Diabetes Care 2013;36:1522-1533.

29. Hashimoto K, Noguchi S, Morimoto Y, et al.: A1C but not serum glycated albumin is elevated in late pregnancy owing to iron deficiency. Diabetes Care 2008;31:1945-1948.

30. Trenti T, Cristani A, Cioni G, et al.: Fructosamine and glycated hemoglobin as indices of glycemic control in patients with liver cirrhosis. Ric Clin Lab 1990;20:261-267.

31. Constanti C, Simo JM, Joven J, Camps J: Serum fructosamine concentration in patients with nephrotic syndrome and with cirrhosis of the liver: the influence of hypoalbuminaemia and hypergammaglobulinaemia. Ann Clin Biochem 1992;29 (Pt 4):437-442.

32. Triger DR, Wright R: Hyperglobulinaemia in liver disease. Lancet 1973;1:1494-1496.

33. Koga M, Saito H, Mukai M, et al.: Serum glycated albumin levels are influenced by smoking status, independent of plasma glucose levels. Acta Diabetol 2009;46: 141-144.

34. Koga M, Murai J, Saito H, et al.: Serum glycated albumin, but not glycated haemoglobin, is low in relation to glycemia in hyperuricemic men. Acta Diabetol 2010;47: 173-177.

35. Koga M, Murai J, Saito H, et al.: Serum glycated albumin, but not glycated hemoglobin, is low in relation to glycemia in men with hypertriglyceridemia. J Diabetes Investig 2010;1:202-207.

36. Koga M, Murai J, Saito H, et al.: Serum glycated albumin levels, but not glycated hemoglobin, is low in relation to glycemia in non-diabetic men with nonalcoholic fatty liver disease with high alanine aminotransferase levels. Clin Biochem 2010;43:1023-1025.

37. Koga M, Otsuki M, Matsumoto S, et al.: Negative association of obesity and its related chronic inflammation with serum glycated albumin but not glycated hemoglobin levels. Clin Chim Acta 2007;378:48-52.
38. Kohzuma T, Yamamoto T, Uematsu Y, et al.: Basic performance of an enzymatic method for glycated albumin and reference range determination. J Diabetes Sci Technol 2011;5:1455-1462.

39. Kohzuma T, Koga M: Lucica GA-L glycated albumin assay kit: a new diagnostic test for diabetes mellitus. Mol Diagn Ther 2010;14:49-51.

40. Juraschek SP, Steffes MW, Selvin E: Associations of alternative markers of glycemia with hemoglobin $\mathrm{A}(1 \mathrm{c})$ and fasting glucose. Clin Chem 2012;58:1648-1655.

41. Jung CH, Hwang YC, Kim KJ, et al.: Development of an HbA1c-based conversion equation for estimating glycated albumin in a Korean population with a wide range of glucose intolerance. PLoS One 2014;9:e95729.

42. Inoue $\mathrm{K}$, Tsujimoto $\mathrm{T}$, Yamamoto-Honda $\mathrm{R}$, et al.: $\mathrm{A}$ newer conversion equation for the correlation between HbA1c and glycated albumin. Endocr J 2014;61:553-560.

43. Tahara Y: Analysis of the method for conversion between levels of HbAlc and glycated albumin by linear regression analysis using a measurement error model. Diabetes Res Clin Pract 2009;84:224-229.

44. Van Dieijen-Visser MP, Seynaeve C, Brombacher PJ: Influence of variations in albumin or total-protein concentration on serum fructosamine concentration. Clin Chem 1986;32:1610.

45. Howey JE, Browning MC, Fraser CG: Assay of serum fructosamine that minimizes standardization and matrix problems: use to assess components of biological variation. Clin Chem 1987;33(2 Pt 1):269-272.

46. Kuenen JC, Borg R, Kuik DJ, et al.: Does glucose variability influence the relationship between mean plasma glucose and HbA1c levels in type 1 and type 2 diabetic patients? Diabetes Care 2011;34:1843-1847.

47. Rodriguez-Segade S, Rodriguez J, Cabezas-Agricola JM, et al.: Progression of nephropathy in type 2 diabetes: the glycation gap is a significant predictor after adjustment for glycohemoglobin (Hb A1c). Clin Chem 2011;57:264-271.

48. Cohen RM, Holmes YR, Chenier TC, Joiner CH: Discordance between HbA1c and fructosamine: evidence for a glycosylation gap and its relation to diabetic nephropathy. Diabetes Care 2003;26:163-167.

49. Buse JB, Freeman JL, Edelman SV, et al.: Serum 1,5anhydroglucitol (GlycoMark): a short-term glycemic marker. Diabetes Technol Ther 2003;5:355-363.

50. Kim WJ, Park CY, Park SE, et al.: Serum 1,5anhydroglucitol is associated with diabetic retinopathy in Type 2 diabetes. Diabet Med 2012;29:1184-1190.

51. Selvin E, Rawlings AM, Grams M, et al.: Association of 1,5-anhydroglucitol with diabetes and microvascular conditions. Clin Chem 2014;60:1409-1418.

52. Takahashi S, Shimada K, Miyauchi K, et al.: Low and exacerbated levels of 1,5-anhydroglucitol are associated with cardiovascular events in patients after first-time elective percutaneous coronary intervention. Cardiovasc Diabetol 2016;15:145.

53. Selvin E, Rawlings A, Lutsey P, et al.: Association of 1,5anhydroglucitol with cardiovascular disease and mortality. Diabetes 2016;65:201-208.

54. Fujiwara T, Yoshida M, Yamada H, et al.: Lower 1,5anhydroglucitol is associated with denovo coronary artery disease in patients at high cardiovascular risk. Heart Vessels 2015;30:469-476.

55. Tetsuo M, Hamada T, Yoshimatsu K, et al.: Serum levels of 1,5-anhydro-D-glucitol during the normal and diabetic 
pregnancy and puerperium. Acta Obstet Gynecol Scand 1990;69:479-485.

56. Nowak N, Skupien J, Cyganek K, et al.: 1,5Anhydroglucitol as a marker of maternal glycaemic control and predictor of neonatal birthweight in pregnancies complicated by type 1 diabetes mellitus. Diabetologia 2013; 56:709-713.

57. Wright LA, Hirsch IB, Gooley TA, Brown Z: 1,5anhydroglucitol and neonatal complications in pregnancy complicated by diabetes. Endocr Pract 2015;21:725-733.

58. Delaney SS, Coley RY, Brown Z: 1,5-Anhydroglucitol: a new predictor of neonatal birth weight in diabetic pregnancies. Eur J Obstet Gynecol Reprod Biol 2015;189:55-58.

59. Balis DA, Tong C, Meininger G: Effect of canagliflozin, a sodium-glucose cotransporter 2 inhibitor, on measurement of serum 1,5-anhydroglucitol. J Diabetes 2014;6:378-380.

60. Pal A, Farmer AJ, Dudley C, et al.: Evaluation of serum 1,5 anhydroglucitol levels as a clinical test to differentiate subtypes of diabetes. Diabetes Care 2010;33:252-257.

61. Miller KM, Beck RW, Bergenstal RM, et al.: Evidence of a strong association between frequency of self-monitoring of blood glucose and hemoglobin A1c levels in T1D exchange clinic registry participants. Diabetes Care 2013;36: 2009-2014.

62. Monnier L, Colette C, Dunseath GJ, Owens DR: The loss of postprandial glycemic control precedes stepwise deterioration of fasting with worsening diabetes. Diabetes Care 2007;30:263-269.

63. Monnier L, Lapinski H, Colette C: Contributions of fasting and postprandial plasma glucose increments to the overall diurnal hyperglycemia of type 2 diabetic patients: variations with increasing levels of $\mathrm{HbA}(1 \mathrm{c})$. Diabetes Care 2003;26:881-885.

64. Riddle M, Umpierrez G, DiGenio A, et al.: Contributions of basal and postprandial hyperglycemia over a wide range of A1C levels before and after treatment intensification in type 2 diabetes. Diabetes Care 2011;34:2508-2514.

65. Standl E, Schnell O, Ceriello A: Postprandial hyperglycemia and glycemic variability: should we care? Diabetes Care 2011;34 Suppl 2:S120-S127.

66. Hanefeld M, Fischer S, Julius U, et al.: Risk factors for myocardial infarction and death in newly detected NIDDM: the Diabetes Intervention Study, 11-year followup. Diabetologia 1996;39:1577-1583.

67. Cavalot F, Pagliarino A, Valle M, et al.: Postprandial blood glucose predicts cardiovascular events and all-cause mortality in type 2 diabetes in a 14-year follow-up: lessons from the San Luigi Gonzaga Diabetes Study. Diabetes Care 2011;34:2237-2243.

68. Ceriello A: Postprandial hyperglycemia and cardiovascular disease: is the HEART2D study the answer? Diabetes Care 2009;32:521-522.

69. Raz I, Ceriello A, Wilson PW, et al.: Post hoc subgroup analysis of the HEART2D trial demonstrates lower cardiovascular risk in older patients targeting postprandial versus fasting/premeal glycemia. Diabetes Care 2011;34: 1511-1513.

70. Raz I, Wilson PW, Strojek K, et al.: Effects of prandial versus fasting glycemia on cardiovascular outcomes in type 2 diabetes: the HEART2D trial. Diabetes Care 2009; 32:381-386.

71. Hill NR, Hindmarsh PC, Stevens RJ, et al.: A method for assessing quality of control from glucose profiles. Diabet Med 2007;24:753-758.
72. Kovatchev BP, Otto E, Cox D, et al.: Evaluation of a new measure of blood glucose variability in diabetes. Diabetes Care 2006;29:2433-2438.

73. Pitsillides AN, Anderson SM, Kovatchev B: Hypoglycemia risk and glucose variability indices derived from routine self-monitoring of blood glucose are related to laboratory measures of insulin sensitivity and epinephrine counterregulation. Diabetes Technol Ther 2011;13:11-17.

74. Kim SK, Kwon SB, Yoon KH, et al.: Assessment of glycemic lability and severity of hypoglycemia in Korean patients with type 1 diabetes. Endocr J 2011;58:433-440.

75. Kohnert KD, Vogt L, Augstein P, et al.: Relationships between glucose variability and conventional measures of glycemic control in continuously monitored patients with type 2 diabetes. Horm Metab Res 2009;41:137-141.

76. Patton SR, Clements MA: Average daily risk range as a measure for clinical research and routine care. J Diabetes Sci Technol 2013;7:1370-1375.

77. Pepper GM, Steinsapir J, Reynolds K: Effect of short-term iPRO continuous glucose monitoring on hemoglobin A1c levels in clinical practice. Diabetes Technol Ther 2012;14: 654-657.

78. Patrascioiu I, Quiros C, Rios P, et al.: Transitory beneficial effects of professional continuous glucose monitoring on the metabolic control of patients with type 1 diabetes. Diabetes Technol Ther 2014;16:219-223.

79. Leinung M, Nardacci E, Patel N, et al.: Benefits of shortterm professional continuous glucose monitoring in clinical practice. Diabetes Technol Ther 2013;15:744-747.

80. Wright L, Vasudevan S, Huynh P, et al. Professional continuous glucose monitoring improves hemoglobin a1c in insulin-requiring diabetic patients. Endocr Rev 2014; 35:MON-1004. http://press.endocrine.org/doi/abs/10.1210/ endo-meetings.2014.DGM.3.MON-1004 (accessed May 7, 2017).

81. Bergenstal RM, Ahmann AJ, Bailey T, et al.: Recommendations for standardizing glucose reporting and analysis to optimize clinical decision making in diabetes: the Ambulatory Glucose Profile (AGP). Diabetes Technol Ther 2013;15:198-211.

82. Brewer KW, Chase HP, Owen S, Garg SK: Slicing the pie. Correlating $\mathrm{HbA}$ - values with average blood glucose values in a pie chart form. Diabetes Care 1998;21:209-212.

83. The relationship of glycemic exposure (HbA1c) to the risk of development and progression of retinopathy in the diabetes control and complications trial. Diabetes 1995;44: 968-983.

84. Qu Y, Jacober SJ, Zhang Q, et al.: Rate of hypoglycemia in insulin-treated patients with type 2 diabetes can be predicted from glycemic variability data. Diabetes Technol Ther 2012;14:1008-1012.

85. Monnier L, Wojtusciszyn A, Colette C, Owens D: The contribution of glucose variability to asymptomatic hypoglycemia in persons with type 2 diabetes. Diabetes Technol Ther 2011;13:813-818.

86. Kovatchev BP, Cox DJ, Gonder-Frederick LA, et al.: Assessment of risk for severe hypoglycemia among adults with IDDM: validation of the low blood glucose index. Diabetes Care 1998;21:1870-1875.

87. Rodbard D: Clinical interpretation of indices of quality of glycemic control and glycemic variability. Postgrad Med 2011;123:107-118.

88. Rodbard D, Bailey T, Jovanovic L, et al.: Improved quality of glycemic control and reduced glycemic variability with 
use of continuous glucose monitoring. Diabetes Technol Ther 2009;11:717-723.

89. Bragd J, Adamson U, Backlund LB, et al.: Can glycaemic variability, as calculated from blood glucose selfmonitoring, predict the development of complications in type 1 diabetes over a decade? Diabetes Metab 2008;34(6 Pt 1):612-616.

90. Soupal J, Skrha J, Jr., Fajmon M, et al.: Glycemic variability is higher in type 1 diabetes patients with microvascular complications irrespective of glycemic control. Diabetes Technol Ther 2014;16:198-203.

91. Snell-Bergeon JK, Roman R, Rodbard D, et al.: Glycaemic variability is associated with coronary artery calcium in men with Type 1 diabetes: the Coronary Artery Calcification in Type 1 Diabetes study. Diabet Med 2010; 27:1436-1442.

92. Kovatchev BP, Cox DJ, Gonder-Frederick LA, Clarke W: Symmetrization of the blood glucose measurement scale and its applications. Diabetes Care 1997;20:1655-1658.

93. Hirsch IB: Glycemic variability: it's not just about A1C anymore! Diabetes Technol Ther 2005;7:780-783.

94. Rodbard D: Hypo- and hyperglycemia in relation to the mean, standard deviation, coefficient of variation, and nature of the glucose distribution. Diabetes Technol Ther 2012;14:868-876.

95. Mazze RS, Strock E, Wesley D, et al.: Characterizing glucose exposure for individuals with normal glucose tolerance using continuous glucose monitoring and ambulatory glucose profile analysis. Diabetes Technol Ther 2008;10:149-159.

96. Mazze RS, Lucido D, Langer O, et al.: Ambulatory glucose profile: representation of verified self-monitored blood glucose data. Diabetes Care 1987;10:111-117.

97. Cryer PE: Hypoglycaemia: the limiting factor in the glycaemic management of type I and type II diabetes. Diabetologia 2002;45:937-948.

98. Zoungas S, Patel A, Chalmers J, et al.: Severe hypoglycemia and risks of vascular events and death. N Engl J Med 2010;363:1410-1418.

99. Seaquist ER, Miller ME, Bonds DE, et al.: The impact of frequent and unrecognized hypoglycemia on mortality in the ACCORD study. Diabetes Care 2012;35:409-414.
100. McCoy RG, Van Houten HK, Ziegenfuss JY, et al.: Increased mortality of patients with diabetes reporting severe hypoglycemia. Diabetes Care 2012;35:1897-1901.

101. Defining and reporting hypoglycemia in diabetes: a report from the American Diabetes Association Workgroup on Hypoglycemia. Diabetes Care 2005;28:1245-1249.

102. International Hypoglycaemia Study Group: Glucose concentrations of less than $3.0 \mathrm{mmol} / \mathrm{L}(54 \mathrm{mg} / \mathrm{dL})$ should be reported in clinical trials: a joint position statement of the American Diabetes Association and the European Association for the Study of Diabetes. Diabetes Care 2017;40:155-157.

103. Bailey T, Bode BW, Christiansen MP, et al.: The performance and usability of a factory-calibrated flash glucose monitoring system. Diabetes Technol Ther 2015;17:787-794.

104. Ish-Shalom M, Wainstein J, Raz I, Mosenzon O: Improvement in glucose control in difficult-to-control patients with diabetes using a novel flash glucose monitoring device. J Diabetes Sci Technol 2016;10:1412-1413.

105. Bolinder J, Antuna R, Geelhoed-Duijvestijn P, et al.: Novel glucose-sensing technology and hypoglycaemia in type 1 diabetes: a multicentre, non-masked, randomised controlled trial. Lancet 2016;388:2254-2263.

106. Haak T, Hanaire H, Ajjan R, et al.: Flash glucose-sensing technology as a replacement for blood glucose monitoring for the management of insulin-treated type 2 diabetes: a multicenter, open-label randomized controlled trial. Diabetes Ther 2016;8:55-73.

107. Bonora B, Maran A, Ciciliot S, et al.: Head-to-head comparison between flash and continuous glucose monitoring systems in outpatients with type 1 diabetes. J Endocrinol Invest 2016;39:1391-1399.

108. Dover AR, Stimson RH, Zammitt NN, Gibb FW: Flash glucose monitoring improves outcomes in a type 1 diabetes clinic. J Diabetes Sci Technol 2017;11(2):442-443.

Address correspondence to: Lorena Alarcon-Casas Wright, MD, FACE University of Washington Medical Center/Roosevelt 4245 Roosevelt Way NE, $3^{\text {rd }}$ Floor Seattle, WA 98105

E-mail: lorenaac@uw.edu 